\title{
A One-Pot Transition-Metal-Free Tandem Process to 1,4-Benzodiazepine Scaffolds
}

Yanqiu Li, ${ }^{\mathrm{a}, 1}$ Chunjing Zhan, ${ }^{\mathrm{a}, 1}$ Bingchuan Yang, ${ }^{\mathrm{a}}$ Xiaoqun Cao, ${ }^{* \mathrm{~b}}$ Chen Ma*a,c

${ }^{a}$ School of Chemistry and Chemical Engineering, Shandong University, Jinan, 250100, P. R. of China

${ }^{b}$ Department of Chemical Engineering, Taishan Medical University, Taian, P. R. of China

c State Key Laboratory of Natural and Biomimetic Drugs, Peking University, Beijing, 100191, P. R. of China Fax +86(531)88564464; E-mail: chenma@sdu.edu.cn

Received: 31.08.2012; Accepted after revision: 16.11 .2012

Abstract: An efficient and practical method for the synthesis of 1,4-benzodiazepines is reported. This methodology offers a transition-metal-free tandem process in one pot. This process is applicable to the construction of a wide variety of 1,4-benzodiazepines and other tricyclic systems with high potential biological and pharmacological activities.

Key words: 1,4-benzodiazepines, pyridazinobenzodiazepines, one-pot reaction, transition-metal-free tandem process

The synthesis of heterocycles ${ }^{2}$ is currently regarded as one of the primary challenges in medicinal chemistry. 1,4Benzodiazepines have received considerable attention because of their biological activities such as antidepressant, ${ }^{3}$ anti-inflammatory, ${ }^{4}$ antagonist, ${ }^{5}$ antimicrobial, ${ }^{6}$ anti-HIV agents, ${ }^{7}$ and antihypertensive ${ }^{8}$ activities. The 1,4-benzodiazepine moiety is also a pivotal intermediate for the production of the medicines, which are currently marketed including olanzapine, clozapine, and viramune (Figure 1). ${ }^{9}$ Pyridazinone derivatives still play an important role in medicinal chemistry owing to their biological activities, ${ }^{10}$ including antimicrobial, ${ }^{11}$ pesticide, ${ }^{12}$ analgesic, ${ }^{13}$ herbicidal, ${ }^{14}$ and anticancer ${ }^{15}$ activities.<smiles>Cc1cc2c(s1)Nc1ccccc1N=C2N1CCN(C)CC1</smiles>

olanzapine

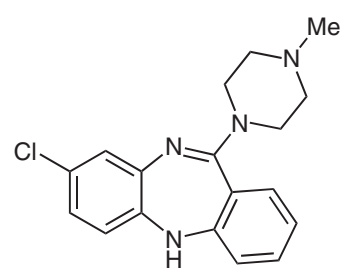

clozapine

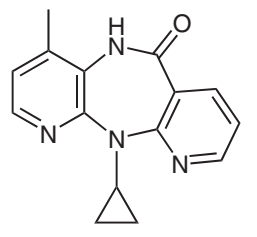

viramune

Figure 1 Structures of olanzapine, clozapine and viramune

SYNTHESIS 2013, 45, 0111-0117

Advanced online publication: 30.11 .2012

DOI: 10.1055/s-0032-1317781; Art ID: SS-2012-H0697-OP

(C) Georg Thieme Verlag Stuttgart · New York
To date, several methods have been reported for the synthesis of these 1,4-benzodiazepine scaffolds. ${ }^{16}$ Wang et al. described the synthesis of these tricyclic compounds in chlorobenzene using ortho-substituted benzoic acids and benzene-1,2-diamine in the presence of copper. ${ }^{17}$ Joshua and co-workers reported the synthesis of a tricyclic lactam by coupling 2,5-dibromonitrobenzene with anthranilic acid via multiple steps. ${ }^{18}$ Diao and Ma developed a new copper-catalyzed strategy to construct these compounds from ortho-substituted aryl bromides and primary amines. ${ }^{19}$ However, these procedures suffer from some disadvantages such as complex manipulation ${ }^{20}$ and the use of transition metal catalyst. ${ }^{21}$ Additionally, to the best of our knowledge, fused pyridazinobenzodiazepines have not been reported. We believe these to be valuable intermediates in medicinal chemistry.

Herein, we report an effective metal-free process for the synthesis of 1,4-benzodiazepines and fused pyridazinobenzodiazepines. Substituted benzenes 2 and N-substituted 2-aminobenzamides $\mathbf{1}$ were used as substrates in the presence of cesium carbonate (Scheme 1). 2-Aminobenzamide (1a) and 4,5-dichloro-2-(tetrahydro-2 $\mathrm{H}$-pyran-2yl)pyridazin-3(2H)-one (4) as substrates, sodium hydride as the base, and DMF as the solvent were used to synthesize the fused pyridazinobenzodiazepine 5 (Scheme 2).<smiles>[R7]c1ccc2c(c1)Nc1ccccc1C(=O)N([R])C2=O</smiles>

Scheme 1 One-pot synthesis of 1,4-benzodiazepines<smiles>CCn1ncc2c(c1=O)NC(=O)c1ccccc1N2</smiles>

Scheme 2 One-pot synthesis of a pyridazinobenzodiazepine 
Initially, the 2-aminobenzamide (1a) and 2,3-difluorobenzonitrile (2e) were chosen as models to determine the optimized conditions. As shown in Table 1, the reaction temperature, base, and solvent were investigated. The preliminary study was conducted using $\mathrm{K}_{2} \mathrm{CO}_{3}$ as the base and DMF as the solvent at room temperature for 48 hours, but the desired product 3d was not obtained. Increasing the temperature to $150{ }^{\circ} \mathrm{C}$ led to the desired product in $30 \%$ yield (Table 1 , entry 2 ). Next, the reaction was carried out using various bases, and $\mathrm{Cs}_{2} \mathrm{CO}_{3}$ provided the highest yields (Table 1, entries 1-4). When strong base such as $\mathrm{NaH}$ or $t$-BuOK was used in the reaction, no desired 3d was obtained (Table 1, entries 1, 4, 7, and 8). Finally, the solvents DMF and DMSO were investigated using $\mathrm{Cs}_{2} \mathrm{CO}_{3}$ as the base (Table 1, entries 3 and 6). To our delight, the desired product $3 \mathrm{~d}$ was obtained in $54 \%$ yield when DMF was used.

The optimized conditions (Table 1, entry 3 ) were applied to a variety of compounds 2 , and the results are summarized in Table 2. As observed in Table 2, the reaction proceeded well with substituted benzenes and $\mathrm{N}$-substituted 2 -aminobenzamides to give a range of 1,4-benzodiazepines. ${ }^{22}$ When 1 was reacted with benzenes containing electron-withdrawing groups (halogen, nitro, cyano, and trifluoromethyl), the corresponding products 3 were generated in moderate to good yields (Table 2, entries 1-6, 8, and 11). Moreover, the reaction was also applied to 2fluoro-1-methyl-3-nitrobenzene (2g) bearing an electrondonating group, affording the desired product $3 \mathrm{f}$ in $67 \%$ yield (Table 2, entry 7). Further, 2-amino- $N$-methylbenz-
Table 1 Reaction Conditions and Yields for the Synthesis of 3d

\begin{tabular}{|c|c|c|c|c|c|}
\hline & & $2 e$ & & & 3d \\
\hline Entry & Base & Solvent & Temp $\left({ }^{\circ} \mathrm{C}\right)$ & Time (h) & Yield (\%) \\
\hline 1 & $\mathrm{NaH}$ & DMF & 150 & 8 & 0 \\
\hline 2 & $\mathrm{~K}_{2} \mathrm{CO}_{3}$ & DMF & 150 & 12 & 30 \\
\hline 3 & $\mathrm{Cs}_{2} \mathrm{CO}_{3}$ & DMF & 150 & 8 & 54 \\
\hline 4 & $t$-BuOK & DMF & 150 & 12 & 0 \\
\hline 5 & $\mathrm{~K}_{2} \mathrm{CO}_{3}$ & DMSO & 150 & 12 & 28 \\
\hline 6 & $\mathrm{Cs}_{2} \mathrm{CO}_{3}$ & DMSO & 150 & 6 & 52 \\
\hline 7 & $t$-BuOK & DMSO & 150 & 12 & 0 \\
\hline 8 & $\mathrm{NaH}$ & DMSO & 150 & 8 & 0 \\
\hline
\end{tabular}

amide (1b) possessing an electron-donating group was reacted with 2e under the same conditions to afford the corresponding product $3 \mathbf{i}$ in excellent yield (Table 2, entry 11).

Table 2 Synthesis of 1,4-Benzodiazepines $3^{\text {a }}$
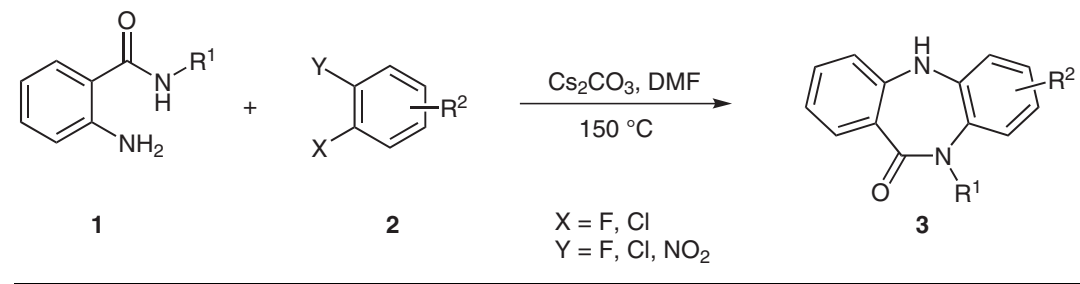

\begin{tabular}{llll}
\hline Entry & $\mathbf{1}$ & $\mathrm{R}^{1}$ & $\mathbf{2}$ \\
\hline & $\mathbf{1 a}$ & $\mathrm{H}$ & $\mathbf{2 a}$
\end{tabular}

$1 \quad \mathbf{1 a} \quad \mathrm{H}$

2a<smiles>O=[N+]([O-])c1cc(F)ccc1F</smiles>

7<smiles>O=[N+]([O-])c1cccc(Cl)c1Cl</smiles>

Time (h)
Yield $(\%)^{\mathrm{b}}$<smiles>O=C1Nc2cc(F)ccc2Nc2ccccc21</smiles> 
Table 2 Synthesis of 1,4-Benzodiazepines $\mathbf{3}^{\text {a }}$ (continued)

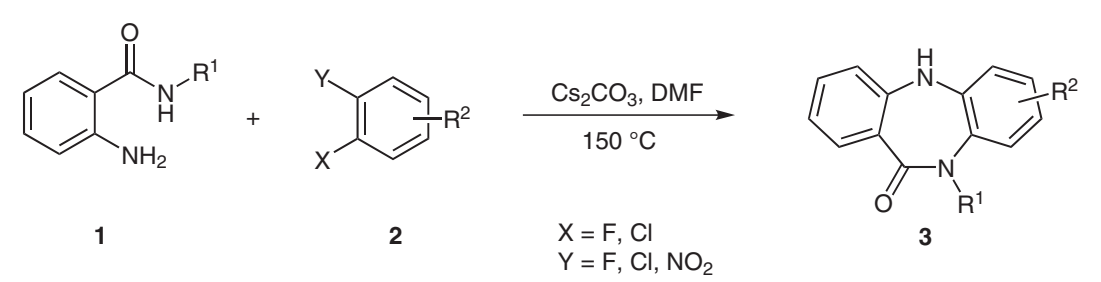

\begin{tabular}{llllll}
\hline Entry & $\mathbf{1}$ & $\mathrm{R}^{1}$ & $\mathbf{2}$ & Time (h) & Product
\end{tabular}

Yield (\%)

$\begin{array}{llll}4 & 1 \mathbf{a} & \mathrm{H}\end{array}$

2d<smiles>N#Cc1ccc(Cl)c([N+](=O)[O-])c1</smiles>

5.5

$3 c$<smiles>N#Cc1ccc2c(c1)NC(=O)c1ccccc1N2</smiles>

6.5<smiles>N#Cc1cccc2c1Nc1ccccc1C(=O)N2</smiles>

$\begin{array}{llll}5 & 1 \mathbf{a} & \mathrm{H}\end{array}$

$2 e$<smiles>N#Cc1cccc(F)c1F</smiles>

2f<smiles>FC(F)(F)c1ccc(Cl)c(Cl)c1</smiles>

6<smiles>O=C1Nc2cc(C(F)(F)F)ccc2Nc2ccccc21</smiles>

5.5<smiles>Cc1cccc2c1Nc1ccccc1C(=O)N2</smiles>

$3 g$<smiles>O=C1Nc2cc(Cl)ccc2Nc2ccccc21</smiles>

6<smiles>O=C1Nc2ccccc2Nc2ccccc21</smiles>

3h<smiles>O=C1Nc2ccccc2Nc2ccccc21</smiles><smiles>CN1C(=O)c2ccccc2Nc2c(C#N)cccc21</smiles>

54

67

60

trace

trace

60<smiles>N#Cc1cccc(F)c1F</smiles>

5

3i

90

${ }^{a}$ Reaction conditions: $1(1 \mathrm{mmol}), 2(1.2 \mathrm{mmol}), \mathrm{Cs}_{2} \mathrm{CO}_{3}(3 \mathrm{mmol}), \mathrm{DMF}(10 \mathrm{~mL}), 150{ }^{\circ} \mathrm{C}, 5-7.5 \mathrm{~h}$.

${ }^{\mathrm{b}}$ Isolated yield. 
As shown in Table 2, the reactant with nitro group (Table 2 , entries 9 and 10) could afford $\mathbf{3 h},{ }^{21,22 a}$ but their yields were much lower than the substrates having a strong electron-donating group. The tricyclic product $\mathbf{3 h}$ was obtained only in trace amounts and was confirmed by HRMS analysis. Conversely, the mono-substituted product 8 was mainly obtained (Scheme 3).<smiles>NC(=O)c1ccccc1N</smiles>

$1 \mathrm{a}$<smiles>[X]c1ccccc1[N+](=O)[O-]</smiles>

$2 \mathbf{i}, \mathbf{j}$

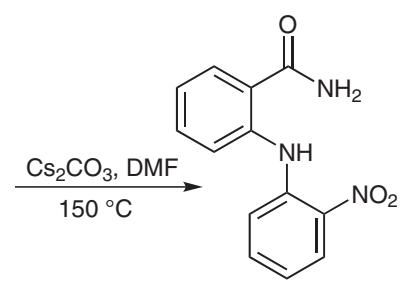

$\mathrm{X}=\mathrm{F}, \mathrm{Cl}$

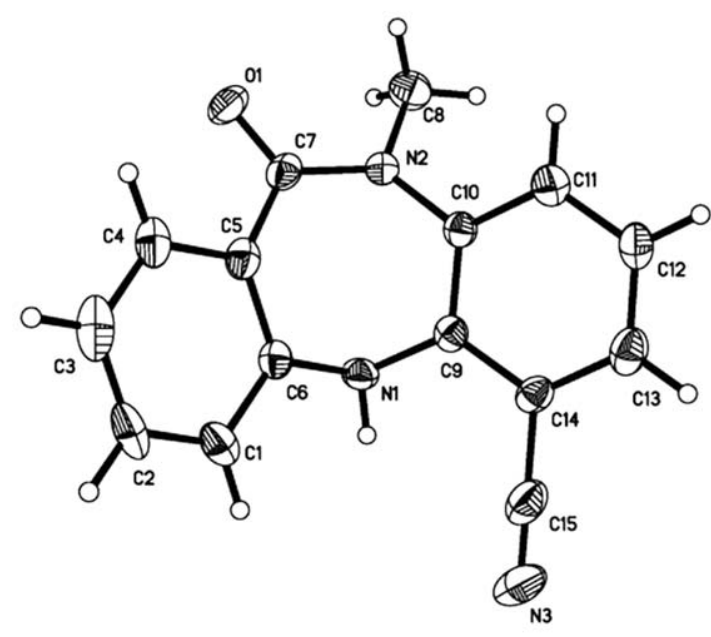

Figure 2 Single crystal X-ray structure of $\mathbf{3 i}$

In summary, we have developed a simple and efficient approach for the synthesis of 1,4-benzodiazepines in moderate to good yields. The prominent features of this methodology are mild and metal catalyst-free reaction conditions. On the basis of the related work, a facile method was discovered for assembling 2-(tetrahydro- $2 \mathrm{H}$-pyran-2yl)-5,11-dihydro- $1 H$-benzo[e]pyridazino[4,5-b][1,4]diazepine-1,10(2H)-dione in high yield. Importantly, this method has high potential uses in the synthesis of biologically relevant compounds. Further investigation for broadening the application of this methodology is currently underway in our laboratory.

2-Amino- $N$-methylbenzamide $(\mathbf{1 b})^{23}$ and 4,5-dichloro-2-(tetrahydro- $2 H$-pyran-2-yl)pyridazin-3 $(2 H)$-one $(4)^{24}$ were prepared according to literature procedures. All other commercial reagents were used without further purification. Petroleum ether (PE) refers to the fraction boiling in the $60-90^{\circ} \mathrm{C}$ range. All the reactions were conducted under $\mathrm{N}_{2}$ atmosphere and monitored by TLC. Melting points were determined on an XD-4 digital micro melting point apparatus. ${ }^{1} \mathrm{H}$ NMR spectra were recorded at $300 \mathrm{MHz}$ on a Bruker Avance 300 spectrometer with TMS as the internal standard and $\mathrm{CDCl}_{3}$ or DMSO- $d_{6}$ as solvent. ${ }^{13} \mathrm{C}$ NMR spectra were obtained on a Bruker Avance $300(75 \mathrm{MHz})$ spectrometer with TMS as the internal standard and $\mathrm{CDCl}_{3}$ or DMSO- $d_{6}$ as solvent. ${ }^{19} \mathrm{~F}$ NMR spectra were obtained in DMSO- $d_{6}$ on a Bruker Avance $300(282 \mathrm{MHz})$ spectrometer. High-resolution mass spectra (HRMS) were obtained on a Q-TOF6510 spectrograph (Agilent). Single crystal X-ray diffraction were performed on a Rigaku RAXIS-SPIDER IP diffractometer at $50 \mathrm{kV}$ and $20 \mathrm{~mA}$ and data collection was performed at 273(2) $\mathrm{K}$ by using graphite monochromated MoK $\alpha$ radiation $(\lambda=$ $0.71073 \AA$ ).

\section{2-Amino- $N$-methylbenzamide (1b)}

To a solution of 2-nitrobenzoic acid $(1.0 \mathrm{~g}, 6 \mathrm{mmol})$ in $\mathrm{MeOH}(15$ $\mathrm{mL}$ ) was slowly added concd $\mathrm{H}_{2} \mathrm{SO}_{4}(2 \mathrm{~mL})$ at r.t. over $15 \mathrm{~min}$. Then, the mixture was refluxed, and the end of the reaction was monitored by TLC (eluent: PE-EtOAc, $5: 1, \mathrm{v} / \mathrm{v}$ ). The reaction mixture was cooled to r.t. Brine $(50 \mathrm{~mL})$ was added and the mixture was extracted with $\mathrm{CH}_{2} \mathrm{Cl}_{2}(2 \times 30 \mathrm{~mL})$. The combined organic phases were dried $\left(\mathrm{MgSO}_{4}\right)$. The solvent was removed under vacuum to afford a residue. A mixture of the residue and aq $\mathrm{MeNH}_{2}(20 \mathrm{~mL})$ was refluxed for $2 \mathrm{~h}$. After cooling, brine $(50 \mathrm{~mL})$ was added and the mixture was extracted with EtOAc $(3 \times 30 \mathrm{~mL})$. The combined organic phases were dried $\left(\mathrm{MgSO}_{4}\right)$ and the solvent was removed un- 
der vacuum to afford an orange solid. The solid was stirred with 12 $\mathrm{M} \mathrm{HCl}(20 \mathrm{~mL})$ at $55^{\circ} \mathrm{C} . \mathrm{SnCl}_{2}(2.84 \mathrm{~g}, 15 \mathrm{mmol})$ was slowly added to the mixture and the resulting solution was stirred at $100{ }^{\circ} \mathrm{C}$ for 15 min. After cooling to r.t., white crystals separated out. The crude product was washed with aq $1 \mathrm{M} \mathrm{NaOH}(30 \mathrm{~mL})$ and extracted with $\mathrm{CH}_{2} \mathrm{Cl}_{2}(2 \times 30 \mathrm{~mL})$. The combined organic phases were dried $\left(\mathrm{MgSO}_{4}\right)$ and concentrated. The crude product was purified by recrystallization from EtOAc $(20 \mathrm{~mL})$ to afford the desired product $\mathbf{1 b}$ as a white solid $(0.62 \mathrm{~g}, 69 \%)$; mp $75.0-76.3{ }^{\circ} \mathrm{C}$ (Lit. ${ }^{23} \mathrm{mp} 76-78$ $\left.{ }^{\circ} \mathrm{C}\right)$.

11-Oxo-10,11-dihydro-5H-dibenzo[b,e $][1,4]$ diazepine-6-carbonitrile (3d); Typical Procedure

To a solution of 2-aminobenzamide (1a; $100 \mathrm{mg}, 0.74 \mathrm{mmol}$ ) in anhyd DMF $(10 \mathrm{~mL})$ were added 2,3-difluorobenzonitrile (2e; 124 $\mathrm{mg}, 0.89 \mathrm{mmol})$ and $\mathrm{Cs}_{2} \mathrm{CO}_{3}(723 \mathrm{mg}, 2.22 \mathrm{mmol})$ at r.t. under a dry $\mathrm{N}_{2}$ atmosphere. The resulting solution was stirred at $150{ }^{\circ} \mathrm{C}$, and the progress of the reaction was monitored by TLC (eluent: PE-EtOAc, $5: 1, \mathrm{v} / \mathrm{v})$. The mixture was diluted with brine $(100 \mathrm{~mL})$ and extracted with EtOAc $(3 \times 30 \mathrm{~mL})$. The combined organic layers were dried $\left(\mathrm{MgSO}_{4}\right)$, filtered, and concentrated in vacuo. Purification of the residue by flash column chromatography (PE-EtOAc, 8:1, v/v) afforded the desired product 3d; yield: $94 \mathrm{mg}$ (54\%); pale yellow crystals; mp $191.8-193.2{ }^{\circ} \mathrm{C}$.

${ }^{1} \mathrm{H}$ NMR $\left(300 \mathrm{MHz}, \mathrm{DMSO}-d_{6}\right): \delta=8.14-8.11(\mathrm{dd}, J=0.9,8.4 \mathrm{~Hz}$, $1 \mathrm{H}), 7.96-7.93(\mathrm{dd}, J=1.5,8.1 \mathrm{~Hz}, 1 \mathrm{H}), 7.89-7.86(\mathrm{dd}, J=0.9$, 8.7 Hz, $1 \mathrm{H}), 7.58-7.52(\mathrm{~m}, 1 \mathrm{H}), 7.37-7.32(\mathrm{~m}, 1 \mathrm{H}), 7.20(\mathrm{~s}, 2 \mathrm{H})$, 6.97-6.94 (dd, $J=0.6,8.4 \mathrm{~Hz}, 1 \mathrm{H}), 6.75-6.70(\mathrm{~m}, 1 \mathrm{H})$.

${ }^{13} \mathrm{C}$ NMR (75 MHz, DMSO- $\left.d_{6}\right): \delta=165.60,150.02,149.16,143.64$, $134.15,129.29,128.91,125.78,116.86,116.44,116.21,116.16$, 105.56, 101.36.

HRMS (ESI): $m / z\left[\mathrm{M}+\mathrm{H}^{+}\right]$calcd for $\mathrm{C}_{14} \mathrm{H}_{9} \mathrm{~N}_{3} \mathrm{O}: 236.0818$; found: 236.0814

8-Fluoro-5H-dibenzo[b,e $][1,4]$ diazepin-11(10H)-one $(3 a)^{21 c}$ Yield: $115 \mathrm{mg}(68 \%)$; orange crystals; $\mathrm{mp} 219.6-222.3^{\circ} \mathrm{C}$ (Lit. ${ }^{21 \mathrm{c}}$ mp 222-223 $\left.{ }^{\circ} \mathrm{C}\right)$.

${ }^{1} \mathrm{H}$ NMR $\left(300 \mathrm{MHz}\right.$, DMSO- $\left.d_{6}\right): \delta=7.90-7.87(\mathrm{dd}, J=1.2,9.3 \mathrm{~Hz}$, $1 \mathrm{H}), 7.81-7.73$ (m, $2 \mathrm{H}), 7.31-7.23(\mathrm{~m}, 2 \mathrm{H}), 7.08$ (s, $2 \mathrm{H}), 6.92$ $6.90(\mathrm{~d}, J=7.5 \mathrm{~Hz}, 1 \mathrm{H}), 6.72-6.66(\mathrm{~m}, 1 \mathrm{H})$.

${ }^{13} \mathrm{C}$ NMR (75 MHz, DMSO- $\left.d_{6}\right): \delta=163.46,159.74\left(\mathrm{~d},{ }^{1} J_{\mathrm{C}, \mathrm{F}}=239.2\right.$ $\mathrm{Hz}), 148.63,148.62\left(\mathrm{~d},{ }^{3} J_{\mathrm{C}, \mathrm{F}}=15.8 \mathrm{~Hz}\right), 137.73,132.60,127.89$, $119.50\left(\mathrm{~d},{ }^{3} J_{\mathrm{C}, \mathrm{F}}=10.5 \mathrm{~Hz}\right), 116.06,115.41,112.18\left(\mathrm{~d},{ }^{2} J_{\mathrm{C}, \mathrm{F}}=24.0\right.$ $\mathrm{Hz}), 106.08\left(\mathrm{~d},{ }^{4} J_{\mathrm{C}, \mathrm{F}}=2.2 \mathrm{~Hz}\right), 98.79\left(\mathrm{~d},{ }^{2} J_{\mathrm{C}, \mathrm{F}}=28.5 \mathrm{~Hz}\right)$.

${ }^{19}$ F NMR (282 MHz, DMSO- $\left.d_{6}\right): \delta=-115.77$.

HRMS (ESI): $m / z\left[\mathrm{M}+\mathrm{H}^{+}\right]$calcd for $\mathrm{C}_{13} \mathrm{H}_{9} \mathrm{FN}_{2} \mathrm{O}: 229.0772$; found: 229.0764 .

6-Chloro-5H-dibenzo $[b, e][1,4]$ diazepin-11(10H)-one $(3 b)^{3,22}$ Yield: $101 \mathrm{mg}(56 \%)$; orange crystals; $\mathrm{mp} 120.9-122.9^{\circ} \mathrm{C}$.

${ }^{1} \mathrm{H}$ NMR $\left(300 \mathrm{MHz}\right.$, DMSO- $\left.d_{6}\right): \delta=7.94-7.91(\mathrm{dd}, J=1.5,8.1 \mathrm{~Hz}$, $1 \mathrm{H}), 7.77-7.74(\mathrm{dd}, J=0.9,8.1 \mathrm{~Hz}, 1 \mathrm{H}), 7.51-7.48(\mathrm{dd}, J=0.9$, $8.1 \mathrm{~Hz}, 1 \mathrm{H}), 7.43-7.41(\mathrm{~d}, J=7.8 \mathrm{~Hz}, 1 \mathrm{H}), 7.38-7.28(\mathrm{~m}, 1 \mathrm{H})$, $7.16(\mathrm{~s}, 2 \mathrm{H}), 6.94-6.92(\mathrm{~d}, J=7.5 \mathrm{~Hz}, 1 \mathrm{H}), 6.73-6.68(\mathrm{~m}, 1 \mathrm{H})$.

${ }^{13} \mathrm{C} \mathrm{NMR}\left(75 \mathrm{MHz}, \mathrm{DMSO}-d_{6}\right): \delta=164.02,149.43,140.78,133.35$, $132.25,129.51,128.51,125.45,120.38,116.62,115.94,111.51$, 106.30 .

HRMS (ESI): $m / z\left[\mathrm{M}+\mathrm{H}^{+}\right]$calcd for $\mathrm{C}_{13} \mathrm{H}_{9} \mathrm{ClN}_{2} \mathrm{O}: 245.0476$; found: 245.0476 .

11-Oxo-10,11-dihydro-5H-dibenzo $[b, e][1,4]$ diazepine-8-carbonitrile (3c)

From 2c, yield: $89 \mathrm{mg}$ (51\%); from 2d, yield: $104 \mathrm{mg}$ (60\%); pale yellow crystals; $\mathrm{mp} 186.1-188.2^{\circ} \mathrm{C}$.

${ }^{1} \mathrm{H}$ NMR $\left(300 \mathrm{MHz}, \mathrm{DMSO}-d_{6}\right): \delta=8.37-8.36(\mathrm{~d}, J=0.9 \mathrm{~Hz}, 1 \mathrm{H})$, 7.95-7.91 (m, 2 H), 7.86-7.82 (dd, $J=1.5,8.4 \mathrm{~Hz}, 1 \mathrm{H}), 7.36-7.30$ $(\mathrm{m}, 1 \mathrm{H}), 7.21(\mathrm{~s}, 2 \mathrm{H}), 6.95-6.92(\mathrm{dd}, J=0.6,8.4 \mathrm{~Hz}, 1 \mathrm{H}), 6.74$ $6.68(\mathrm{~m}, 1 \mathrm{H})$.

${ }^{13} \mathrm{C}$ NMR (75 MHz, DMSO- $d_{6}$ ): $\delta=166.12,150.05,148.48,145.72$, $134.10,129.66,128.85,120.43,119.42,116.80,116.07,115.39$, 107.03, 105.60.

HRMS (ESI): $m / z\left[\mathrm{M}+\mathrm{H}^{+}\right]$calcd for $\mathrm{C}_{14} \mathrm{H}_{9} \mathrm{~N}_{3} \mathrm{O}: 236.0818$; found: 236.0839 .

\section{8-(Trifluoromethyl)-5H-dibenzo $[b, e][1,4]$ diazepin-11(10H)-one} $(3 \mathrm{e})^{21 \mathrm{c}}$

Yield: $97 \mathrm{mg}$ (47\%); white crystals; $\mathrm{mp} 205.1-207.0^{\circ} \mathrm{C}$ (Lit. ${ }^{21 \mathrm{c}} \mathrm{mp}$ 200-202 $\left.{ }^{\circ} \mathrm{C}\right)$.

${ }^{1} \mathrm{H}$ NMR $\left(300 \mathrm{MHz}\right.$, DMSO- $\left.d_{6}\right): \delta=9.94(\mathrm{~s}, 1 \mathrm{H}), 7.96-7.94(\mathrm{~d}, J=$ $2.7 \mathrm{~Hz}, 1 \mathrm{H}), 7.92(\mathrm{~s}, 1 \mathrm{H}), 7.77-7.72(\mathrm{~m}, 2 \mathrm{H}), 7.27-7.22(\mathrm{~m}, 1 \mathrm{H})$, 6.80-6.77 (d, $J=7.2 \mathrm{~Hz}, 1 \mathrm{H}), 6.64-6.60(\mathrm{~m}, 1 \mathrm{H}), 6.46(\mathrm{~s}, 1 \mathrm{H})$.

${ }^{13} \mathrm{C}$ NMR (75 MHz, DMSO- $d_{6}$ ): $\delta=167.56,150.18,139.28,132.81$, $128.85,128.84,127.52,126.64\left(\mathrm{q},{ }^{2} J_{\mathrm{C}, \mathrm{F}}=32.2 \mathrm{~Hz}\right), 126.50(\mathrm{q}$, $\left.{ }^{3} J_{\mathrm{C}, \mathrm{F}}=3.75 \mathrm{~Hz}\right), 123.41\left(\mathrm{q},{ }^{1} J_{\mathrm{C}, \mathrm{F}}=270.8 \mathrm{~Hz}\right), 124.43\left(\mathrm{q},{ }^{3} J_{\mathrm{C}, \mathrm{F}}=3.38\right.$ $\mathrm{Hz}), 116.81,114.97,113.52$.

${ }^{19} \mathrm{~F}$ NMR (282 MHz, DMSO- $\left.d_{6}\right): \delta=-60.75$.

HRMS (ESI): $m / z\left[\mathrm{M}+\mathrm{H}^{+}\right]$calcd for $\mathrm{C}_{14} \mathrm{H}_{9} \mathrm{~F}_{3} \mathrm{~N}_{2} \mathrm{O}: 279.0740$; found: 279.1576 .

\section{6-Methyl-5H-dibenzo[b,e][1,4]diazepin-11(10H)-one (3f)}

Yield: $111 \mathrm{mg}(67 \%)$; orange crystals; $\mathrm{mp} 136.1-138.2{ }^{\circ} \mathrm{C}$.

${ }^{1} \mathrm{H}$ NMR (300 MHz, DMSO- $\left.d_{6}\right): \delta=11.26(\mathrm{~s}, 1 \mathrm{H}), 8.72(\mathrm{~s}, 1 \mathrm{H})$, $8.19-8.16(\mathrm{~d}, J=8.7 \mathrm{~Hz}, 1 \mathrm{H}), 7.66-7.63(\mathrm{~d}, J=8.1 \mathrm{~Hz}, 1 \mathrm{H})$, $7.33-7.26$ (dd, $J=7.5,15.6 \mathrm{~Hz}, 1 \mathrm{H}), 7.01-6.98(\mathrm{~d}, J=8.4 \mathrm{~Hz}, 1$ $\mathrm{H}), 6.79-6.73(\mathrm{dd}, J=7.5,11.7 \mathrm{~Hz}, 2 \mathrm{H}), 5.78(\mathrm{~s}, 1 \mathrm{H}), 2.48(\mathrm{~s}, 3 \mathrm{H})$.

${ }^{13} \mathrm{C}$ NMR (75 MHz, DMSO- $d_{6}$ ): $\delta=168.00,149.94,147.95,135.49$, $134.47,133.59,127.47,125.97,124.04,122.01,117.79,117.19$, 114.77, 22.22 .

HRMS (ESI): $m / z$ [M $\left.+\mathrm{H}^{+}\right]$calcd for $\mathrm{C}_{14} \mathrm{H}_{12} \mathrm{~N}_{2} \mathrm{O}: 225.1022$; found: 225.1032 .

8-Chloro-5H-dibenzo[b,e $][1,4]$ diazepin-11(10H)-one (3g) $)^{3,22}$ Yield: $108 \mathrm{mg}(60 \%)$; pale yellow crystals; $\mathrm{mp} 231.2-232.8^{\circ} \mathrm{C}$ (Lit. ${ }^{3} \mathrm{mp} 232-233^{\circ} \mathrm{C}$ ).

${ }^{1} \mathrm{H}$ NMR $\left(300 \mathrm{MHz}\right.$, DMSO- $\left.d_{6}\right): \delta=7.95-7.94(\mathrm{dd}, J=2.1,8.4 \mathrm{~Hz}$, $1 \mathrm{H}), 7.91-7.88(\mathrm{dd}, J=1.5,8.1 \mathrm{~Hz}, 1 \mathrm{H}), 7.80-7.77(\mathrm{~d}, J=8.4 \mathrm{~Hz}$, $1 \mathrm{H}), 7.46-7.42(\mathrm{dd}, J=2.1,8.4 \mathrm{~Hz}, 1 \mathrm{H}), 7.33-7.27(\mathrm{~m}, 1 \mathrm{H}), 7.14$ (s, $2 \mathrm{H}), 6.94-6.91(\mathrm{dd}, J=0.6,8.4 \mathrm{~Hz}, 1 \mathrm{H}), 6.72-6.67(\mathrm{~m}, 1 \mathrm{H})$.

${ }^{13} \mathrm{C}$ NMR $\left(75 \mathrm{MHz}\right.$, DMSO- $\left.d_{6}\right): \delta=164.02,149.43,140.78,133.35$, $132.25,129.51,128.51,125.45,120.38,116.62,115.94,111.51$, 106.30 .

HRMS (ESI): $m / z\left[\mathrm{M}+\mathrm{H}^{+}\right]$calcd for $\mathrm{C}_{13} \mathrm{H}_{9} \mathrm{ClN}_{2} \mathrm{O}: 245.0476$; found: 245.0480 .

10-Methyl-11-oxo-10,11-dihydro-5H-dibenzo $[b, e][1,4]$ diazepine-9-carbonitrile (3i)

Yield: $166 \mathrm{mg}$ (90\%); white crystals; mp $141.5-143.0{ }^{\circ} \mathrm{C}$.

${ }^{1} \mathrm{H}$ NMR $\left(300 \mathrm{MHz}, \mathrm{DMSO}-d_{6}\right): \delta=7.92-7.89(\mathrm{dd}, J=1.5,7.8 \mathrm{~Hz}$, $1 \mathrm{H}), 7.42-7.35(\mathrm{~m}, 3 \mathrm{H}), 7.20-7.17(\mathrm{dd}, J=8.1 \mathrm{~Hz}, 1 \mathrm{H}), 7.14-7.08$ $(\mathrm{m}, 1 \mathrm{H}), 6.99-6.96(\mathrm{dd}, J=0.9,8.1 \mathrm{~Hz}, 1 \mathrm{H}), 6.06(\mathrm{~s}, 1 \mathrm{H}), 3.53$ (s, $3 \mathrm{H})$.

${ }^{13} \mathrm{C}$ NMR (75 MHz, DMSO- $d_{6}$ ): $\delta=169.39,157.31,153.99,143.20$, $131.41,128.73,126.62,123.83,120.62,120.35,119.24,118.42$, $115.01,108.46,26.16$.

HRMS (ESI): $m / z\left[\mathrm{M}+\mathrm{H}^{+}\right]$calcd for $\mathrm{C}_{15} \mathrm{H}_{11} \mathrm{~N}_{3} \mathrm{O}$ : 250.0975; found: 250.0993 .

2-[(2-Nitrophenyl)amino]benzamide (8)

From 2i, yield: $113 \mathrm{mg}(60 \%)$; from $\mathbf{2 j}$, yield: $104 \mathrm{mg}(55 \%)$; orange crystals; $\mathrm{mp} 201.3-203.2^{\circ} \mathrm{C}$. 
${ }^{1} \mathrm{H}$ NMR (300 MHz, DMSO- $\left.d_{6}\right): \delta=11.22(\mathrm{~s}, 1 \mathrm{H}), 8.14-8.11(\mathrm{dd}$, $J=1.2,8.4 \mathrm{~Hz}, 2 \mathrm{H}), 7.75-7.72(\mathrm{dd}, J=1.2,7.8 \mathrm{~Hz}, 1 \mathrm{H}), 7.60-7.43$ (m, $5 \mathrm{H}), 7.15-7.10(\mathrm{~m}, 1 \mathrm{H}), 7.02-6.96(\mathrm{~m}, 1 \mathrm{H})$.

${ }^{13} \mathrm{C} \mathrm{NMR}\left(75 \mathrm{MHz}, \mathrm{DMSO}-d_{6}\right): \delta=170.00,139.65,139.26,135.91$, $135.53,131.41,129.13,126.25,124.39,122.23,120.05,119.47$, 117.88 .

HRMS (ESI): $m / z\left[\mathrm{M}+\mathrm{H}^{+}\right]$calcd for $\mathrm{C}_{13} \mathrm{H}_{11} \mathrm{~N}_{3} \mathrm{O}_{3}: 258.0834$; found: 258.0886 .

\section{2-(Tetrahydro-2H-pyran-2-yl)-5,11-dihydro-1H-benzo[e $[e]$ pyr-} idazino $[4,5-b][1,4]$ diazepine-1,10 $(2 H)$-dione $(5)$

To a solution of 2 -aminobenzamide $(\mathbf{1 a} ; 100 \mathrm{mg}, 0.74 \mathrm{mmol})$ in anhyd DMF $(10 \mathrm{~mL})$ were added 4,5-dichloro-2-(tetrahydro- $2 H$-pyran-2-yl)pyridazin-3(2H)-one $(4 ; 196 \mathrm{mg}, 0.89 \mathrm{mmol})$ and $\mathrm{NaH}$ $(60 \%, 89 \mathrm{mg}, 2.22 \mathrm{mmol})$ at r.t. under a dry $\mathrm{N}_{2}$ atmosphere: The mixture was stirred for $2 \mathrm{~h}$ at $80^{\circ} \mathrm{C}$ and then quenched with brine $(100 \mathrm{~mL})$. The precipitated crude product was purified by recrystallization from EtOAc $(20 \mathrm{~mL})$ to afford the desired product 5 as an orange solid; yield: $194 \mathrm{mg}$ (84\%); orange crystals; mp 271.8 $273.2^{\circ} \mathrm{C}$.

${ }^{1} \mathrm{H}$ NMR $\left(300 \mathrm{MHz}, \mathrm{DMSO}-d_{6}\right): \delta=9.81(\mathrm{~s}, 1 \mathrm{H}), 7.72-7.69(\mathrm{dd}$, $J=1.0,9.2 \mathrm{~Hz}, 2 \mathrm{H}), 7.61-7.66(\mathrm{~s}, 1 \mathrm{H}), 7.33-7.29(\mathrm{~m}, 1 \mathrm{H}), 7.11-$ $7.09(\mathrm{~d}, J=8.0 \mathrm{~Hz}, 1 \mathrm{H}), 6.87-6.83(\mathrm{dd}, J=7.6,15 \mathrm{~Hz}, 1 \mathrm{H}), 5.84$ $5.81(\mathrm{dd}, J=1.9,10.8 \mathrm{~Hz}, 1 \mathrm{H}), 3.96-3.93(\mathrm{~d}, J=11.0 \mathrm{~Hz}, 1 \mathrm{H})$, 3.61-3.54 (m, $1 \mathrm{H}), 2.10-2.00(\mathrm{~m}, 1 \mathrm{H}), 1.94-1.91(\mathrm{~d}, J=12.0 \mathrm{~Hz}$, $1 \mathrm{H}), 1.71-1.60$ (m, $2 \mathrm{H}), 1.51-1.46$ (m, $2 \mathrm{H})$.

${ }^{13} \mathrm{C} \mathrm{NMR}\left(75 \mathrm{MHz}\right.$, DMSO- $d_{6}$ ): $\delta=167.11,156.02,146.05,134.84$, $133.64,132.88,128.67,122.62,121.68,120.72,120.50,83.22$, $67.92,28.35,25.12,22.75$.

HRMS (ESI): $m / z\left[\mathrm{M}+\mathrm{H}^{+}\right]$calcd for $\mathrm{C}_{16} \mathrm{H}_{16} \mathrm{~N}_{4} \mathrm{O}_{3}: 313.1295$; found: 313.1291 .

\section{Acknowledgment}

We are grateful to the National Science Foundation of China (No. 21172131) and the State Key Laboratory of Natural and Biomimetic Drugs of Peking University (No. K20090205) for financial support of this research.

Supporting Information for this article is available online at http://www.thieme-connect.com/ejournals/toc/synthesis.

\section{References}

(1) These authors contributed equally to this work.

(2) (a) Monro, A. M.; Quinton, R. M.; Wrigley, T. I. J. Med. Chem. 1963, 6, 255. (b) Huang, A.; Liu, F.; Zhan, C.; Liu, Y.; Ma, C. Org. Biomol. Chem. 2011, 9, 7351. (c) Liu, Y.; Chu, C.; Huang, A.; Zhan, C.; Ma, Y.; Ma, C. ACS Comb. Sci. 2011, 13, 547.

(3) (a) Capuano, B.; Crosby, I. T.; Lloyd, E. J.; Taylor, D. A. Aust. J. Chem. 2002, 55, 565. (b) Liao, Y.; DeBoer, P.; Meier, E.; Wikström, H. J. Med. Chem. 1997, 40, 4146.

(4) Coyne, W. E.; Cusic, J. W. J. Med. Chem. 1967, 10, 541.

(5) (a) Hasvold, L. A.; Wang, L.; Przytulinska, M.; Xiao, Z.; Chen, Z.; Gu, W.-Z.; Merta, P. J.; Xue, J.; Kovar, P.; Zhang, H.; Park, C.; Sowin, T. J.; Rosenberg, S. H.; Lin, N.-H. Bioorg. Med. Chem. Lett. 2008, 18, 2311. (b) Murugesan, N.; Gu, Z.; Lee, V.; Webb, M. L.; Liu, E. C.-K.; Hermsmeier, M.; Hunt, J. T. Bioorg. Med. Chem. Lett. 1995, $5,253$.

(6) Thuston, D. E.; Bose, D. S. Chem. Rev. 1994, 94, 433.

(7) (a) Breslin, H. J.; Kukla, M. J.; Ludovici, D. W.; Mohrbacher, R.; Ho, W.; Miranda, M.; Rodgers, J. D.; Hitchens, T. K.; Leo, G.; Gauthier, D. A.; Ho, C. Y.; Scott,
M. K.; De Clercq, E.; Pauwels, R.; Andries, K.; Janssen, M. A. C.; Janssen, P. A. J. Med. Chem. 1995, 38, 771. (b) Kukla, M. J.; Breslin, H. J.; Diamond, C. J.; Grous, P. P.; Ho, C. Y.; Miranda, M.; Rodgers, J. D.; Sherrill, R. G.; De Clercq, E.; Pauwels, R.; Andries, K.; Moens, L. J.; Janssen, M. A. C.; Janssen, P. A. J. J. Med. Chem. 1991, 34, 3187.

(8) Albright, J. D.; Feich, M. F.; Santos, E. G. D.; Dusza, J. P.; Sum, F. W.; Venkatesan, A. M.; Coupet, J.; Chan, P. S.; Ru, X.; Mazandarani, H.; Bailey, T. J. Med. Chem. 1998, 41, 2442.

(9) (a) Capuano, B.; Crosby, I. T.; McRobb, F. M.; Podloucka, A.; Taylor, D. A.; Vom, A.; Yuriev, E. Aust. J. Chem. 2010, 63, 116. (b) Matloubi, H.; Ghandi, M.; Zarrindast, M.-R.; Saemian, N. Appl. Radiat. Isot. 2001, 55, 789.

(c) Sasikumar, T. K.; Burnett, D. A.; Zhang, H.; SmithTorhan, A.; Fawzi, A.; Lachowicz, J. E. Bioorg. Med. Chem. Lett. 2006, 16, 4543. (d) Hunziker, F.; Kunzle, F.; Schmutz, J.; Schindler, O. Helv. Chim. Acta 1964, 47, 1163. (e) Hunziker, F.; Fischer, E.; Schmutz, J. Helv. Chim. Acta 1967, 50, 1588. (f) Schmutz, J.; Kunzle, F.; Hunziker, F.; Gauch, R. Helv. Chim. Acta 1967, 50, 245. (g) Liao, Y.; Venhuis, B. J.; Rodenhuis, N.; Timmerman, W.; Wikström, H. J. Med. Chem. 1999, 42, 2235.

(10) (a) Sridhara, A. M.; Reddy, K. R. V.; Keshavayya, J.; Goud, P. S. K.; Somashekar, B. C.; Bose, P.; Peethambar, S. K.; Gaddam, S. K. Eur. J. Med. Chem. 2010, 45, 4983. (b) Prime, M. E.; Courtney, S. M.; Brookfield, F. A.; Marston, R. W.; Walker, V.; Warne, J.; Boyd, A. E.; Kairies, N. A.; Saal, W.; Limberg, A.; Georges, G.; Engh, R. A.; Goller, B.; Rueger, P.; Rueth, M. J. Med. Chem. 2011, 54, 312. (c) Del, Olmo. E.; Barboza, B.; Ybarra, M. I.; LopezPerez, J. L.; Carron, R.; Sevilla, M. A.; Boselli, C.; San, Feliciano. A. Bioorg. Med. Chem. Lett. 2006, 16, 2786. (d) Napoletano, M.; Norcini, G.; Pellacini, F.; Marchini, F.; Morazzoni, G.; Ferlenga, P.; Pradella, L. Bioorg. Med. Chem. Lett. 2000, 10, 2235. (e) Ma, C.; Liu, S. J.; Xin, L.; Falck, J. R.; Shin, D. S. Tetrahedron 2006, 62, 9002. (f) Cho, S. D.; Song, S. Y.; Park, Y. D.; Kim, J. J.; Joo, W. H.; Shiro, M.; Falck, J. R.; Shin, D. S.; Yoon, Y. J. Tetrahedron Lett. 2003, 44, 8995. (g) Ma, C.; Cho, S. D.; Falck, J. R.; Shin, D. S. Synth. Commun. 2004, 34, 1399. (h) Liu, Y.; Ma, Y.; Zhan, C.; Huang, A.; Ma, C. Synlett 2012, 23, 255.

(i) Huang, A.; Qiao, Z.; Zhang, X.; Yu, W.; Zheng, Q.; Ma, Y.; Ma, C. Tetrahedron 2012, 68, 906.

(11) Sayed, G. H.; Sayed, M. A.; Mahmoud, M. R.; Shaaban, S. S. Egypt. J. Chem. 2002, 45, 767; Chem. Abstr. 2003, 141, 243501.

(12) Piaz, V. D.; Ciciani, G.; Giovannoni, M. P. Synthesis 1994, 669.

(13) Katrusiak, B.; Unlu, S.; Banoglu, E.; Kupeli, E.; Yesilada, E.; Sahin, M. F. Arch. Pharm. (Weinheim, Ger.) 2003, 336, 406.

(14) Verhelst, T.; Verbeeck, S.; Ryabtsova, O.; Depraetere, S.; Maes, B. U. W. Org. Lett. 2011, 13, 272.

(15) (a) Svete, J. J. Heterocycl. Chem. 2005, 42, 361. (b) Schwartz, A.; Beke, G.; Kovári, Z.; Böcskey, Z.; Farkas, Ö.; Mátyus, P. J. Mol. Struct. 2000, 528, 49.

(16) (a) Nardi, M.; Cozza, A.; De Nino, A.; Oliverio, M.; Procopio, A. Synthesis 2012, 44, 800. (b) Tiberghien, A. C.; Evans, D. A.; Kiakos, K.; Martin, C. R. H.; Hartley, J. A.; Thurston, D. E.; Howard, P. W. Bioorg. Med. Chem. Lett. 2008, 18, 2073. (c) Beccalli, E. M.; Broggini, G.; Paladino, G.; Penoni, A.; Zoni, C. J. Org. Chem. 2004, 69, 5627. (d) Okubo, T.; Yoshikawa, R.; Chaki, S.; Okuyama, S.; Nakazato, A. Bioorg. Med. Chem. 2004, 12, 3569.

(17) Wang, L.; Sullivan, G. M.; Hexamer, L. A.; Hasvold, L. A.; Thalji, R.; Przytulinska, M.; Tao, Z.-F.; Li, G.; Chen, Z.; 
Xiao, Z.; Gu, W.-Z.; Xue, J.; Bui, M.-H.; Merta, P.; Kovar, P.; Bouska, J. J.; Zhang, H.; Park, C.; Stewart, K. D.; Sham, H. L.; Sowin, T. J.; Rosenberg, S. H.; Lin, N.-H. J. Med. Chem. 2007, 50, 4162.

(18) Joshua, A. V.; Sharma, S. K.; Strelkov, A.; Scott, J. R.; Martin-Iverson, M. T.; Abrams, D. N.; Silverstone, P. H.; McEwan, A. J. B. Bioorg. Med. Chem. Lett. 2007, 17, 4066.

(19) Diao, X.; Xu, L.; Zhu, W.; Jiang, Y.; Wang, H.; Guo, Y.; Ma, D. Org. Lett. 2011, 13, 6422.

(20) (a) Su, J.; Tang, H.; McKittrick, B. A.; Burnett, D. A.; Zhang, H.; Smith-Torhan, A.; Fawzi, A.; Lachowicz, J. Bioorg. Med. Chem. Lett. 2006, 16, 4548. (b) Zhao, H.-Y.; Liu, G. J. Comb. Chem. 2007, 9, 1164. (c) Elmore, C. S.; Dorff, P. N.; Heys, J. R. J. Label. Compd. Radiopharm 2010, 53, 787. (d) Al-Tel, T. H.; Al-Qawasmeh, R. A.; Schmidt, M. F.; Al-Aboudi, A.; Rao, S. N.; Sabri, S. S.; Voelter, W. J. Med. Chem. 2009, 52, 6484. (e) Broggini, G.; Marchi, I. D.; Martinelli, M.; Paladino, G.; Penoni, A. Lett. Org. Chem. 2004, 1, 221. (f) Hanze, A. R.; Strube, R. E.; Greig, M. E. J. Med. Chem. 1963, 6, 767. (g) Beccalli, E. M.; Broggini, G.; Paladino, G.; Zoni, C. Tetrahedron 2005, 61, 61.

(h) Zhang, L.; Meier, W.; Wats, E.; Costello, T. D.; Ma, P.;
Ensinger, C. L.; Rodgers, J. M.; Jacobson, I. C.; Rajagopalan, P. Tetrahedron Lett. 1995, 36, 8387.

(21) (a) Levy, O.; Erez, M.; Varon, D.; Keinan, E. Bioorg. Med. Chem. Lett. 2001, 11, 2921. (b) Basolo, L.; Beccalli, E. M.; Borsini, E.; Broggini, G.; Khansaa, M.; Rigamonti, M. Eur. J. Org. Chem. 2010, 9, 1694. (c) Tsvelikhovsky, D.; Buchwald, S. L. J. Am. Chem. Soc. 2011, 133, 14228. (d) Lu, S.-M.; Alper, H. J. Am. Chem. Soc. 2005, 127, 14776.

(e) Bunce, R. A.; Schammerhorn, J. E. J. Heterocycl. Chem. 2006, 43, 1031. (f) Tardibono, L. P.; Miller, M. J. Org. Lett. 2009, 11, 1575. (g) Majumdar, K. C.; Ganai, S. Synlett 2011, 1881.

(22) (a) Leyva-Pérez, A.; Cabrero-Antonino, J. R.; Corma, A. Tetrahedron 2010, 66, 8203. (b) Binaschi, M.; Boldetti, A.; Gianni, M.; Maggi, C. A.; Gensini, M.; Bigioni, M.; Parlani, M.; Giolitti, A.; Fratelli, M.; Valli, C.; Terao, M.; Garattini, E. ACS Med. Chem. Lett. 2010, $1,411$.

(23) (a) Tu, T.; Wang, Z.; Liu, Z.; Feng, X.; Wang, Q. Green Chem. 2012, 14, 921. (b) Mahiwal, K.; Kumar, P.; Narasimhan, B. Med. Chem. Res. 2012, 21, 293.

(24) Ma, C.; Liu, S-J.; Xin, L.; Falck, J. R.; Shin, D-S. Tetrahedron 2006, 62, 9002. 